\title{
UM NOVO MODELO DE SISTEMAS DE INFORMAÇÃO PARA INSTITUIÇÕES DE P\&D
}

\author{
Nilce Chaves Gattaz \\ Rejane Contow
}

\begin{abstract}
Resumo
Propõe uma nova abordagem de sistemas de informação para instituições de $P \& D$, concorrentemente a uma mudança de paradigma. Enfoca a interação e integração com os diversos segmentos da sociedade como forma de monitorar as demandas de informação e viabilizar o atendimento dessas demandas. Apresenta uma metodologia de implantação e o modelo do sistema de informação em desenvolvimento no CNPMA/ EMBRAPA. Enfatiza as formas de coleta e disseminação de informações em tempo real com a participação dos geradores dessas informações.
\end{abstract}

\section{Palavras-Chave}

Sistema de Informação; Redes de Comunicação de Dados; Instituições de P\&D; Bases de Dados; Correio Eletrônico; Grupos de Interesse; Cadeia Produtiva.

\section{INTRODUÇÃO}

As instituições públicas de pesquisa e desenvolvimento têm como premissa sua inserção na sociedade. Portanto, têm como finalidade gerar, adquirir, organizar, armazenar, processar, recuperar e disseminar informações conforme as necessidades da sociedade.

A cadeia de pesquisa e desenvolvimento tem como pontos principais: a identificação e análise de problemas, a elaboração de projetos, a sua execução, a análise e a síntese da informação gerada, o acompanhamento da utilidade dessa informação e seus efeitos diretos e indiretos na sociedade.

Na identificação de problemas de pesquisa, é essencial a participação das comunidades produtivas, consumidoras e científicas, em tempo real para que o exercício de análise e identificação dos problemas seja uma tarefa do dia-a-dia. Para isso, o uso das redes de comunicação de dados é uma atividade imprescindível pois favorece a intercomunicação dos indivíduos das diversas comunidades.

A intercomunicação apenas através de documentos inibe a participação da sociedade em tempo real no exercício da pesquisa, pois o período entre a geração da informação e a sua recepção através de documentos é, na maioria das vezes, longo, diminuindo assim o valor real da informação gerada.

Entretanto, é essencial que o conhecimento disponível seja indexado e que a informação esteja organizada para o trabalho de recuperação e processamento, pois a sistematização e o processamento da informação evita, muitas vezes, a duplicidade de experimentação e, portanto, garante o uso mais eficaz e eficiente dos recursos públicos. A atividade de processamento da informação aflora as lacunas do conhecimento, gerando incertezas que delinearão os 
experimentos - veículo de geração de nova informação.

Desta forma, quanto mais informações organizadas e acessíveis de conhecimento em disponibilidade, melhor será a qualidade dos experimentos e do reúso da informação na resolução de problemas.

Durante a elaboração de projetos, que reflitam a resolução de problemas previamente identificados, o conhecimento disponível indexado torna-se uma variável preponderante, determinando na metodologia o desenho da solução.

A execução, a análise e o acompanhamento dos projetos de pesquisa exigem uma realimentação em tempo real dos bancos de dados, colocando, assim, à disposição da sociedade a competência da instituição de pesquisa.

Assim sendo, este trabalho apresenta uma metodologia para modelar um Sistema de Informação, discutindo os princípios que regem os problemas mencionados; a definição e desenvolvimento do modelo do Sistema de Informação; e apresentando um modelo em desenvolvimento, cujos resultados têm chegado, em tempo real, às comunidades científicas e consumidoras, e seus "feedbacks" muito têm contribuído para o sucesso do desenvolvimento dos projetos de pesquisa.

Este trabalho visa, portanto, contribuir para a transformação das instituições de P\&D como agentes de agregação de informação e de formulação de mudanças com a qualidade requerida pelos diversos setores da sociedade.

\section{CARACTERIZAÇÃO DO SISTEMA DE INFORMAÇÃO}

\subsection{Identificação do Problema}

Os sistemas de informação, de um modo geral, são ainda muito voltados para a informação documental. Mesmo apresentando uma evolução no conceito (Biblioteca x Sistemas de Informação), a evolução não vai muito além do conceito. Os planos e ações não apresentam grandes mudanças isto porque a tendência tem sido introduzir novas tecnologias em procedimentos tradicionais e muitas vezes ultrapassados.

Uma sociedade em constante evolução, surpreendida dia a dia por fatos novos, exige, no entanto, forçosamente uma recontextualização dos elementos com ela envolvida. É necessário abandonar, muitas vezes, os princípios e procedimentos organizacionais e criar outros inteiramente inovadores. Essa realidade não difere quando se trata de discutir sistemas de informação. Ao contrário, está totalmente dependente. No entanto, quando se procura identificar estudos nesta área, muitas são as preocupações que advem dos enfoques em que eles se centralizam.

O trabalho de VASCONCELOS (1994), por exemplo, avalia a satisfação da demanda dos usuários do Centro de Tecnologia da UFCE, utilizando um modelo de nível de satisfação para medir a disponibilidade de documentos. O primeiro aspecto a destacar é quanto ao nível de esforço requerido para desenvolver estudos voltados para o enfoque "disponibilidade de documentos" quando se propõe discussões sobre Transferência de Informação no limiar do ano 2000. O outro, não menos importante, diz respeito à revisão de literatura desse trabalho que variou entre 1971 e 1980. Este dado por si só evidencia o descompasso entre estudos de demandas de informação e necessidades de informação. Se levarmos em consideração as significativas mudanças ocorridas no macroambiente nesta última década, com certeza, não poderemos chegar às mesmas conclusões. Esse novo macroambiente favorece o estabelecimento de um sistema acelerado de criação de riquezas que está, cada vez mais, dependente da troca de dados, informações e conhecimento (FURLAN, 1994) e, portanto, a preocupação com o nível de satisfação não pode enfocar tão somente "disponibilidade de documentos".

O trabalho de COSTA \& SILVA (1994) relata as etapas conduzidas no processo de implantação da Gestão pela Qualidade Total onde o problema fundamental encontrado foi "solicitações não atendidas". Na Fig. 6, onde se encontra a identificação dos produtos e serviços, o conteúdo não apresenta nenhuma evolução. Ali encontra-se: 1.Consulta/Orientação; 2. Empréstimo de Publicações/Documentos; 3. Comutação; 4. Circulação de Periódico; 5. Pesquisa Bibliográfica; 6. Tradução e Versões; 7. Relatório Gerencial do SEDOT e 8. 
Planejamento, Coordenação e Supervisão. Conforme citam os autores "com base na identificação dos produtos, clientes e fornecedores, foi elaborado o Macrofluxo de Processos. O Macrofluxo é uma ferramenta fundamental para o conhecimento das atividades desenvolvidas, suas inter-relações, os produtos resultantes e o relacionamento com clientes e fornecedores, permitindo assim, o desdobramento em fluxogramas de cada processo interno, bem como a avaliação da eficácia do sistema (SEDOT)."

Percebe-se nestes dois trabalhos uma tendência a sistemas de produção pré-definidos que não contemplam as mudanças de mercado, isto é, sistemas que não são capazes de captar, em tempo real, o ruído e se ajustar às mudanças de rumo, de atuar num contexto altamente competitivo que exige uma batalha interminável entre concorrentes. Conforme diz PORTER (1992) "mesmo longos períodos de estabilidade podem ser abruptamente terminados por movimentos competitivos". Esta afirmação é totalmente pertinente na conjuntura atual. Mesmo assim, as conclusões dos trabalhos comentados não propõem mudanças que poderiam vir a reverter esse quadro.

O trabalho de OLIVEIRA (1994) vem reforçar a afirmação de que os Sistemas de Informação são ainda muito voltados para a informação documental. Conforme a autora, apesar da importância da informação no processo industrial como insumo na produção, tanto quanto a matéria e a energia, há uma crença generalizada de que a informação, ou os trabalhos oferecidos por serviços e centros de informação, se referem apenas a livros e a serviços de bibliotecas tradicionais. Ainda, conforme OLIVEIRA (1994), "não existe uma Política de Informação em Pernambuco (e esta afirmação pode ser estendida a nível nacional)* que dê respaldo à transferência de tecnologia industrial". As novas demandas da sociedade pós-industrial estão exigindo uma mudança de paradigma das instituições, o que vai refletir diretamente nos Sistemas de Informação, não havendo, portanto, mais condições de manter-se estruturas tradicionais como as ainda existentes. Estas estruturas são incapazes de incorporar as influências contextuais por serem mecânicas e burocráticas, ao contrário do que o mundo pósindustrial está impondo, ou seja, uma estrutura

\footnotetext{
* Comentário das autoras.
}

mais dinâmica, cujos sistemas orgânicos são capazes de perceber os impactos sobre suas próprias atividades.

A proposta do Sistema Público de Acesso à Bases de Dados - SPA, conforme MIRANDA (1994) é também muito relevante no entendimento de uma nova proposta de Sistemas de Informação. Segundo o autor, o "SPA envolve riscos que precisam ser detectados e minimizados a tempo através de uma adequada e permanente análise de sistema, em busca de melhores alternativas para a solução de seus problemas operacionais e conjunturais emergentes". O desafio do SPA é poder atender às condições de mudança e adaptar-se às tensões externas para garantir o status quo no mercado, mercado esse em constante ebulição. Reforçando o enfoque que estamos tentando levantar nestes comentários, MIRANDA (1994) ainda diz: "infelizmente, porém, até que o número de postos de serviço configure uma rede ampla de oportunidades, considerando inclusive o acesso através de laboratórios próprios e domicílios dos usuários, deve transcorrer um espaço de tempo nada desprezível. E com um agravante: até lá os usuários experimentarão canais próprios; para isto concorrem a proliferação de redes do tipo correio eletrônico, com possibilidades amplas de consultas entre os pares (via colégios invisíveis, através do correio eletrônico) a nível internacional, quase sem custos diretos; a proliferação de edições de bases de dados em $\mathrm{CD}$-Rom que continuarão chegando às melhores bibliotecas concorrentes do SPA e, quem sabe, a possibilidade futura de acesso mais econômico às bases originais, no Exterior, onde presumivelmente estão mais atualizadas, e em maior número do que aquelas oferecidas pelo SPA".

O caso do SPA, assim como muitos outros, inclusive o da própria EMBRAPA, parece se enquadrar na afirmação de LOVEMAN em seu artigo na Business Week (TECHNOLOGY... 1993) de que os ganhos advêm da capacidade de suportar rupturas e não da tecnologia. E isto vem ocorrendo porque os planejamentos de Sistemas de Informação continuam sendo ainda enfocados em documentos e não em informação, longe de poder atuar no contexto de uma sociedade pósindustrial. $\mathrm{O}$ erro fundamental é procurar ver a tecnologia através das lentes dos processos existentes.

Conforme HAMMER \& CHAMPY (1994), tentar corrigir os erros através da 
reformulação das partes individuais do processo é a melhor forma que se conhece de garantir a continuidade do desempenho deficiente. Ainda conforme HAMMER \& CHAMPY (1994) ao invés de se perguntar como podemos aproveitar essas novas capacidades tecnológicas para melhorar ou otimizar o que fazemos atualmente, dever-se-ia perguntar como podemos aproveitar a tecnologia para fazer aquilo que ainda não estamos fazendo.

Esta afirmação de HAMMER \& CHAMPY (1994) parece contribuir também para as discussões sobre a formação dos profissionais bibliotecários face à questão da transferência de informação apresentadas por BREGLIA \& RODRIGUES (1994), quando afirmam: “...entende-se que só através de uma nova orientação metodológica que privilegie a pesquisa qualitativa, com a geração de novas linhas temáticas que não apresentem dicotomia com o contexto, e principalmente com uma mudança de rumo na análise, alargando o campo visual dos observadores, com o olhar voltado para todos os elementos envolvidos no processo educadores e educandos - é possível chegar-se à formação de recursos humanos com competência suficiente para atuar na indústria de transferência de informação".*

O que se percebe é uma necessidade imperiosa de mudança de um perfil profissional; porém nesse enfoque apresentado, ainda continuamos com uma dicotomia com o contexto. Os educadores e educandos estão longe de ser todos os elementos do processo. Precisase urgentemente modelar o processo, identificar os elementos que interagem e interferem nesse processo e aí ter-se-á a visão de que a formação de recursos humanos para a área de informação depende de uma noção mais abrangente de processo. Técnicas geradoras de estoques, conforme são denominadas por MIRANDA (1990) a catalogação, a classificação, a indexação (com seus vocabulários controlados) e a recuperação, precisam ser repensadas no atual contexto de uso de informação. Caso contrário, segundo FURLAN (1994), não teremos muito tempo para decidir entre fazer parte do rolo compressor ou fazer parte da estrada. Esta é a imposição que se coloca na era da tecnologia da informação ao profissional da informação.

Talvez o exemplo dado pela ABAG (1993) sobre o número de elos que podem ser

* Grifo das autoras. enumerados na complexa produção atual de OVOS possa ajudar a esclarecer ainda mais nossa análise. Como diz a ABAG (1993) "nada aparentemente mais natural que encontrá-lo em bandejas nos supermercados. É como se, para estar ali, classificado por tamanho, cor e qualidade, o ovo não tivesse requerido mais esforço que o de transportá-lo da granja para a prateleira". Somente a compreensão do que significa o investimento em pesquisa e desenvolvimento nas áreas de genética, nutrição, manejo, defesa sanitária, equipamentos para a produção, embalagem, entre outros intermináveis elos, dá conta, em parte, de como é complexa a produção atual de OVOS.

Sem esta mudança de enfoque, e sem o olhar realmente voltado para todos os elementos do processo, o conceito de indústria de transferência de informação que, segundo BREGLIA \& RODRIGUES (1994) significa comunicar, difundir o conhecimento estocado, e não mais a informação estocada, ainda continuará ineficiente e incapaz de permitir a modelagem de Sistemas de Informação intensivos em informação e contextuais. Estará, isto sim, favorecendo a modelagem de Sistemas de Informação com uma massa de dados padronizados que não mais atendem às necessidades de informação.

\subsection{O Sistema e seus Princípios}

Os elementos e as regras de transformação de um sistema identificam o seu comportamento, sua complexidade e a sua pertinência a uma classe de problemas (SIMON, 1973). A informação em tempo real tem volatibilidade alta e necessita de contínua reformatação. A mudança freqüente dos processos das cadeias produtivas conforme as evoluções tecnológicas e o comportamento concorrente de seus elos, e a redefinição contínua dos papéis dos agentes participantes das instituições de P\&D caracterizam essa classe de sistema como fracamente estruturados (SOBRINHO, 1991). Isto é, a interligação entre os elementos do sistema é dependente do contexto e está em constante mudança, causando assim uma indefinição da sua estrutura e, portanto, fazendo com que as suas próprias soluções interfiram na formulação dos seus problemas no tempo.

Como se pode notar na Fig. 1, supondo-se uma inovação tecnológica (como a 
telecomunicação digital), uma nova restrição legislativa (como a lei de agrotóxicos), uma descoberta genética vegetal (com variedades resistentes a uma série de pragas), um novo modelo econômico (como o Plano Real), entre outras constantes mudanças na sociedade não controláveis pela instituição de pesquisa, ter-seá uma estrutura indefinida das interligações existentes entre os elos da cadeia produtiva.
A interdependência da cadeia de problemas e soluções explica também o comportamento do sistema (Fig. 2), isto é, o uso indevido de agrotóxicos e de mecanização agrícola para uma certa variedade pode solucionar o problema 1 , porém, pode causar o problema 2 , e assim por diante no mesmo espaço e tempo em que as mudanças da sociedade estão ocorrendo.

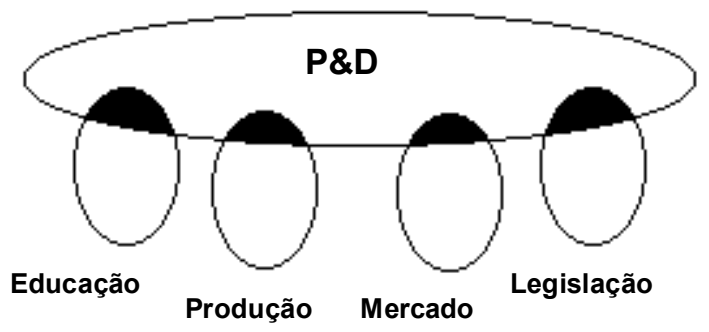

FIG. 1. Cadeia Produtiva

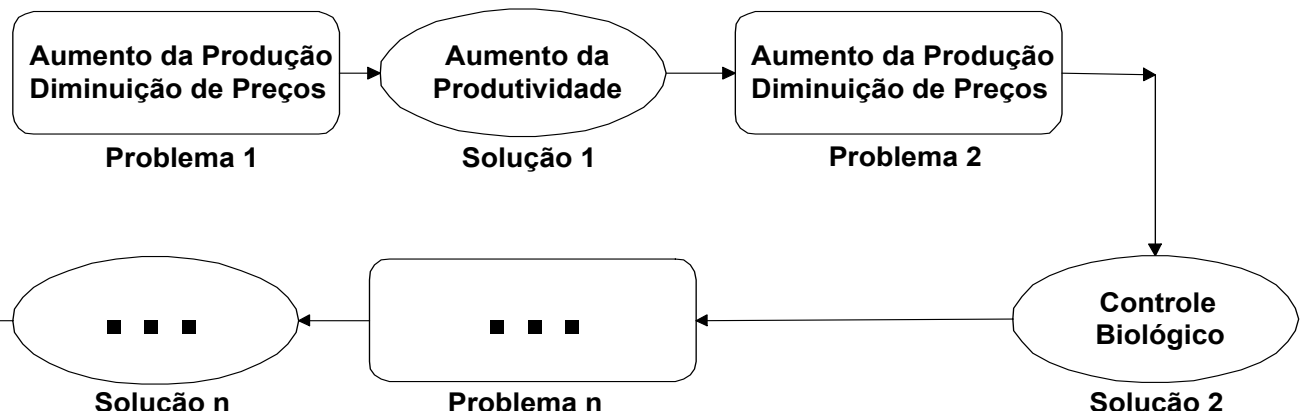

FIG.2. Cadeia de Interdependências de Problemas e Soluções

A problemática desses sistemas de informação, que buscam satisfazer e evoluir o contexto, pode ser delineada como se segue:

a) não existem regras que estabeleçam se a solução é completa;

b) os sintomas e as causas se confundem no espaço;

c) a formulação exaustiva e definitiva do problema, em geral, não é possível; e

d) a cadeia de interdependência entre os problemas e as suas soluções não permitem tratamento ou formulação separadas.

A abordagem mais adequada para a modelagem de tais sistemas é regida pelos seguintes princípios (YEH, 1991): 


\section{Inclusão}

Sistemas são construídos por uma equipe multidisciplinar que representem a cadeia dos seus usuários.

\section{Separação de Assuntos}

Dividir e conquistar, enfocando sobre a subdivisão e decomposição, almejando estabilidade funcional para lidar com a complexidade.

\section{Coevolução}

Formular os problemas relacionando-os com os critérios de desenho das soluções, procurando tratar os efeitos colaterais de dependência.

\section{Protoiteração}

Prototipar a interação entre o sistema e o contexto, buscando antecipar o comportamento co-evolutivo das mudanças.

\section{Melhoria Contínua}

Desenvolver medidas de eficiência, eficácia e efetividade, de forma que sejam permanentemente aplicadas para garantir a qualidade do reúso.

\section{Reificação}

Elaborar a base do julgamento explícito e comunicar as razões e fundamento lógico através de trocas de informação e/ou discussões públicas no sistema.

A abordagem das instituições de $\mathrm{P} \& \mathrm{D}$, sobre os seus sistemas de informação, é, em geral, a clássica e tem como premissa que os sistemas independem do contexto, não permitindo a volatibilidade da informação e sua disponibilidade em tempo real. Procuram indexar, prioritariamente, informações documentais e mais especificamente bibliográficas, ou seja, dados secundários publicados. Não existe um sistema de informação da pesquisa que integre a sua instituição aos outros agentes da cadeia produtiva e tão pouco com as outras instituições parceiras da pesquisa. Mais preocupante ainda é o desfacelamento das informações e das ações integradas dentro das suas próprias instituições geradoras de informação.

As informações de pesquisa, bibliográficas e administrativas estão, na maioria das instituições, dispersas e não disponíveis para acesso público no sistema, o que dificulta o reúso da informação e promove tanto uma descontinuidade nos processos como uma duplicação de esforços, deteriorando a imagem da instituição e distanciando-a cada vez mais da sociedade.

No sentido de facilitar a reengenharia das instituições de $\mathrm{P} \& \mathrm{D}$, para o atendimento dos anseios da sociedade, descreve-se, na seção seguinte, uma proposta de modelo e metodologia para a sua implantação.

\section{MODELO DE SISTEMA DE INFORMAÇÃO}

\subsection{Definição do modelo}

O modelo de sistema de informação, conforme Fig. 3, apresentada na página ao lado, consiste na definição de quatro elementos básicos: banco de dados, equipe multidisciplinar, grupos de interesse e tecnologia de informação (SUNDGREN, 1975).

\section{Banco de Dados}

A definição de banco de dados consiste na identificação e caracterização das variáveis dos processos das cadeias produtivas onde a instituição de P\&D é parte integrante. Essas variáveis devem refletir a realidade do contexto em que a instituição está inserida, considerando os aspectos educacionais, científicos e tecnológicos, produtivos, mercadológicos e legais.

Os bancos de dados são, então, identificados e/ou estruturados (quando não disponíveis) através de grupos de variáveis cujos valores caracterizam os possíveis interesses situacionais e definam os estados de transição do sistema de informação. Desta forma os bancos de dados existem conforme o contexto, em tempo real, e são flexíveis conforme a necessidade situacional da instituição. Os bancos de dados constituem, portanto, a infra-estrutura flexível para mudanças e passam a ser essenciais para as reengenharias requeridas pelos esforços e posicionamentos da instituição de $\mathrm{P} \& \mathrm{D}$ no mercado.

\section{Equipe Multidisciplinar}

A equipe multidisciplinar consiste de profissionais que, em conjunto, possam aconselhar e definir problemáticas situacionais 


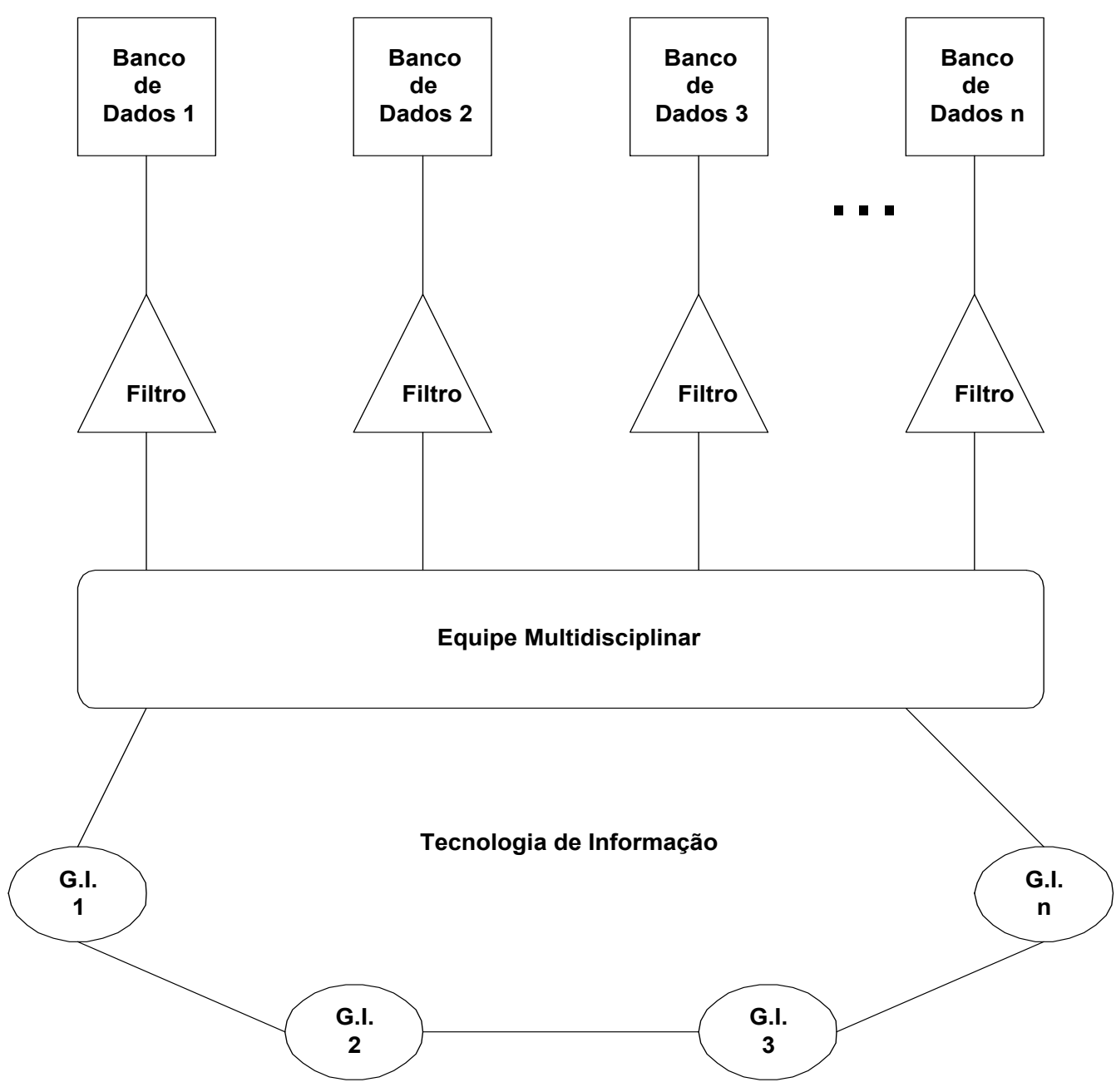

FIG. 3 Modelo de Sistema de Informação

da instituição. Essa equipe define propostas de empreendimentos e de políticas da instituição, utilizando-se dos bancos de dados, através dos "filtros" que traduzem o formato e extraem a informação requerida. Nesse sentido a equipe deve ser representativa do contexto situacional da sociedade em que se insere a instituição, sendo, desse modo, autoridade para simular e experimentar as situações atuais e desejáveis.

\section{Grupos de Interesse}

Um grupo de interesse consiste de grupos de pessoas que comungam o mesmo problema e que, permanentemente, discutem as idéias em tempo real, através de um veículo de comunicação que satisfaça a realidade.

Os grupos de interesse são elementos que zelam pela "transpiração" do modelo perante a sociedade. As "ignorâncias" e "sabedorias" da instituição se espelham nos estados das discussões dos diversos grupos, provocando assim a reciclagem e recapacitação (quando necessária) dos recursos humanos sem traumas para a instituição, pois cada técnico participante se auto-avalia e toma o seu caminho orientado pelo contexto, que está explícito pelos próprios participantes, e assim, reificando o sistema. O modus-operandi dos grupos de interesse contribui 
para uma maior interação entre as equipes das diversas instituições, integrando assim esforços paralelos para a resolução dos problemas.

\section{Tecnologia de Informação}

Esse elemento do modelo consiste de uma plataforma de software e aplicações que permitam o uso de métodos, técnicas e ferramentas em telecomunicação, computação, multimídia e processos de forma integrada. Essa tecnologia deve capacitar o redesenho dos bancos de dados e dos empreendimentos propostos, a reformulação de políticas, as mudanças de protocolos de comunicação e os redesenhos dos processos das cadeias produtivas. Isto é, a tecnologia que permite a reengenharia do próprio modelo, garantindo assim a sua evolução contínua, e sem traumas, no sentido de acomodar novos desafios culturais, sócio-econômicos e tecnológicos.

\subsection{Metodologia}

A metodologia para o desenvolvimento de sistemas de informação tem como característica o uso de técnicas participativas e interdisciplinares na análise e implementação de cada uma das suas ações. Neste sentido, o desenvolvimento do sistema de informação deverá contar com equipes de pesquisadores, assistentes de pesquisa, profissionais de informática e de informação.

À equipe de pesquisadores cabe indicar as necessidades de conteúdo das bases de dados de acordo com os seus resultados de pesquisa e com as necessidades da sociedade. Aos profissionais de informação cabe a definição do formato do banco de dados, a sua alimentação e o plano de capacitação, voltado para pesquisadores e assistentes de pesquisa, quanto à alimentação e à recuperação das informações. À equipe de informática cabe desenhar e especificar o sistema e, juntamente com os profissionais de informação, manter-se atualizada quanto ao uso das mais recentes tecnologias de informação, visando o constante aperfeiçoamento do sistema.

O sistema de informação compõe-se de vários bancos de dados para permitir que as instituições de P\&D coletem as necessidades/ demandas da sociedade. Objetivam favorecer a análise e delineamento dos projetos de pesquisa, bem como tornar disponíveis as informações e tecnologias geradas. Neste sentido, torna-se imprescindível priorizar o desenvolvimento dos bancos de dados de Problemas - Temas Projetos de Pesquisa - Pesquisadores e Instituições - Informação Bibliográfica Instrumentos e Objetos de Pesquisa nos Domínios Específicos da Instituição.

Os grupos de interesse, segundo a sua definição, podem ser constituídos, aplicando-se as seguintes regras:

Cada líder de projeto cria uma lista de discussão sobre seu respectivo assunto de pesquisa, através das redes de comunicação de dados e institui para ela um moderador.

$\mathrm{O}$ moderador conduz as discussões e periodicamente apresenta um relatório sobre os resultados atingidos.

O profissional de informação organiza as informações que compõem os relatórios periódicos e dissemina-os através do banco de dados de temas.

A tecnologia de informação adequada para essa classe de problemas deve conter necessariamente sistemas de redes compatíveis com todos os protocolos das redes públicas de comunicação de dados, um sistema de "software" para a caracterização e simulação de processos, um sistema de prototipagem e integração de aplicações e um sistema de armazenamento, processamento e recuperação de informação.

É recomendável também que a instituição tenha convênios de cooperação técnica com outros centros/serviços de informação para facilitar a coleta e intercâmbio de informações.

\section{EXEMPLO DE UM SISTEMA DE INFORMAÇÃO}

Nesta seção descreve-se o Sistema de Informação em desenvolvimento no Centro Nacional de Pesquisa de Monitoramento e Impacto Ambiental na Agricultura - CNPMA/ EMBRAPA - composto dos bancos de dados a seguir descritos (Fig. 4).

Os bancos de dados que fazem parte do sistema estão em fase de estruturação e prototipagem para posterior validação e alimentação e são flexíveis para mudanças na sua estrutura, conforme a necessidade de introdução 


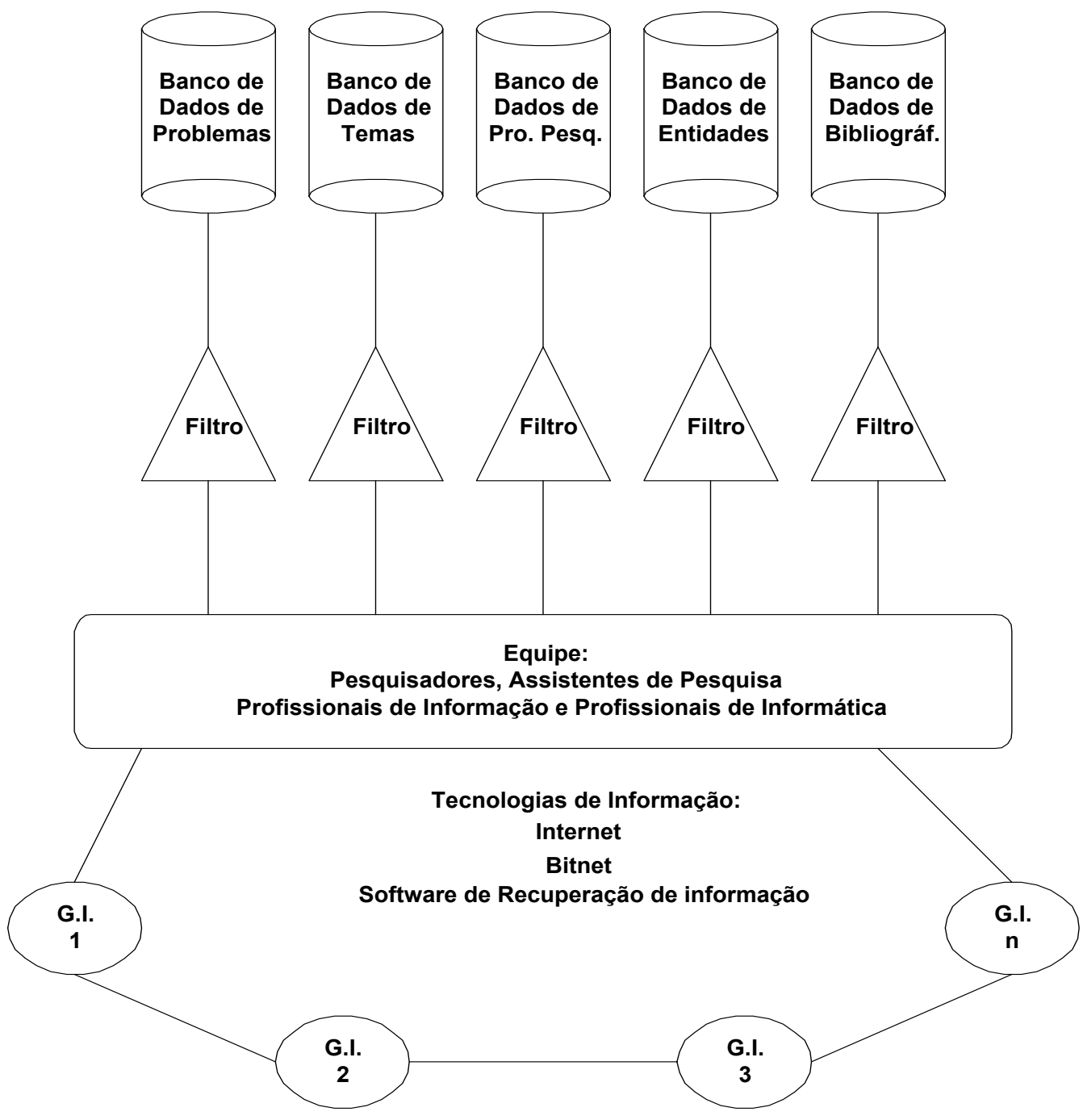

FIG. 4. Sistema de Informação do CNPMA/EMBRAPA

de novas informações. São alimentados periodicamente com informações fornecidas pelos líderes de projeto e por informações coletadas em outras fontes de acesso.

\subsection{Bancos de Dados}

\section{Banco de Dados de Problemas}

Armazena o levantamento dos problemas advindos dos vários segmentos da sociedade, tais como: produtores, associações de classe, cooperativas, instituições de ensino e pesquisa, entre outros. Este levantamento é feito através da interação com estes segmentos, usando os vários meios de comunicação existentes. Estes problemas são analisados e classificados de acordo com a sua complexidade e necessidade de solução. Os menos complexos são transformados em temas para debates entre os participantes dos grupos de interesse para se tentar obter resultados/soluções a curto prazo. Os mais complexos servirão de subsídio para a tomada de decisão na priorização de projetos de pesquisa e, da mesma forma, serão debatidos com os grupos de interesse para 
intercâmbio de conhecimento e, conseqüentemente, agilização dos resultados.

\section{Banco de Dados de Temas}

Disponibiliza os resultados e/ou soluções das discussões dos temas discutidos pelos grupos de interesse, organizados pelo moderador da lista e pelos profissionais de informação.

\section{Bancos de Dados de Pesquisadores e Instituições}

Torna disponíveis nomes, endereços, áreas de atuação, resumos de projetos de pesquisa em desenvolvimento, entre outros dados, de pesquisadores e instituições nacionais e internacionais que atuam nas áreas de interesse de pesquisa do Centro. É interessante enfatizar que a coleta e a atualização destas informações é feita através de questionários enviados e recebidos via redes de comunicação de dados.

\section{Bancos de Dados de Projetos de Pesquisa}

Disponibiliza os resultados de pesquisas em andamento, bem como os resultados alcançados através dos projetos de pesquisa do Centro. Neste banco são incluídas não só informações textuais, mas também na forma gráfica, apresentando mapas, audiovisuais, e outros, por serem informações integrantes dos projetos.

\section{Bancos de Dados Bibliográficos}

Torna disponíveis informações documentais existentes no Centro relativas à sua missão. São incluídos neste banco os livros, os periódicos, as teses, os folhetos, as separatas, as obras de referências, os mapas, o material audiovisual, entre outros.

\subsection{Equipe}

A equipe envolvida no desenvolvimento do sistema é multidisciplinar e é constituída de pesquisadores com a função de líderes de projeto, com formação em Agronomia, Biologia, Química, Ecologia, Matemática e Estatística; e de técnicos em Análise de Sistemas e Ciência da Informação.

\subsection{Tecnologia de Informação}

As ferramentas usadas pelo sistema para a coleta, armazenamento e disseminação das informações são o correio eletrônico e o World Wide Web (WWW).

OWWW é um software desenvolvido pelo "European Particle Physics Laboratory", da Suíça, composto por um cliente e um servidor. O cliente acessa um servidor através de híper texto e busca qualquer documento da rede independentemente de onde ele esteja e da forma como ele está disponível, interagindo com as várias ferramentas de software existentes para redes. Além de textos, pode-se recuperar imagens e sons. Qualquer cliente Web de qualquer máquina acessa anonimamente qualquer servidor Web, também instalado em qualquer máquina na Internet. O WWW é um software de domínio público, podendo ser recuperado através da Internet, existindo vários tipos de clientes e servidores, adequados aos diferentes equipamentos disponíveis (Mosaic e Netscape para ambientes gráficos, e Lynx para ambientes não gráficos), (OLIVEIRA, 1995).

O correio eletrônico é uma ferramenta de comunicação de dados entre indivíduos e/ou comunidades que tem objetivos comuns. Através desta ferramenta, os grupos de interesse se integram, discutem idéias, e trocam arquivos relativos a assuntos técnico-científicos, em tempo real.

\subsection{Grupos de Interesse}

Coloca disponíveis temas de pesquisa para discussão em redes de comunicação de dados, visando um esforço cooperativo de desenvolvimento de projetos de pesquisa. A discussão dos temas é feita de forma organizada, onde um grupo de pesquisadores da comunidade científica e/ou produtiva contribui com seus conhecimentos para um determinado projeto de pesquisa. Há um moderador que conduz as discussões, convergindo-as para um relatório final e posterior divulgação através do banco de dados de temas.

Devido à limitação de equipamentos de informática existentes no Centro, foi criado apenas um grupo de interesse na área de controle biológico e seu impacto no meio ambiente. $\mathrm{O}$ 
grupo de interesse conta, atualmente, com 400 pesquisadores, oriundos de 40 países, incluindo o Brasil, podendo, dia a dia, aumentar o número de inscritos nesse grupo.

Após o termino da instalação dos novos equipamentos adquiridos, serão criados novos grupos de interesse para discussão de temas sobre as demais áreas de atuação do Centro.

\section{CONCLUSÃO}

Conforme discutido nesse trabalho, esta abordagem vem contribuir com uma nova visão da instituição de P\&D, evidenciando-a, na sua competência, como centro de referência e agente de transformação na sociedade, através da sua interação e integração com os demais segmentos da cadeia produtiva, explicitando a valoração, integridade e validade da informação no espaço, em tempo real.

Sugere-se, portanto, que os profissionais de informação adotem uma postura mais proativa e, juntamente com os pesquisadores, desenvolvam modelos similares para sistematizar o armazenamento, processamento, geração e disseminação da informação nas suas respectivas instituições.

\section{REFERÊNCIAS BIBLIOGRÁFICAS}

BREGLIA, V. L. A., RODRIGUES, M. E. F. A formação dos profissionais bibliotecários e a questão da transferência de informação. In: CONGRESSO LATINO-AMERICANO DE BIBLIOTECONOMIA E DOCUMENTAÇ $\tilde{A} O, 2$. CONGRESSO BRASILEIRO DE BIBLIOTECONOMIA E DOCUMENTAČ̃̃O, 17. Anais. Belo Horizonte: ABMG/Esc. Bibl. UFMG, 1994. p. $395-414$

COSTA, M. F. T. de, LIMA, E. B. de. Implantação da Gestão pela Qualidade Total - GQT na Seção de Documentação Técnica do Distrito de Exploração do Sudeste (DESUD) - Petrobrás. In: CONGRESSO LATINO-AMERICANO DE BIBLIOTECONOMIA

DOCUMENTAÇÃO, 2. CONGRESSO BRASILEIRO DE BIBLIOTECONOMIA E DOCUMENTACC̃̃O, 17. Anais. Belo Horizonte: ABMG/Esc. Bibl. UFMG, 1994.

\section{p. $69-80$}

FURLAN, J. D. Reengenharia da Informação; do mito à realidade. São Paulo: Makron Books, 1994. 132p.

GATTAZ, N. C. et alii. Estabelecimento de centro de referência em informação sobre monitoramento e impacto ambiental na agricultura. Jaguariúna: EMBRAPA/ CNPMA, 1994. 12p. (EMBRAPA. Programa 14.0.94.221)

HAMMER, M., CHAMPY, J. Reengenharia; revolucionando a empresa em função dos clientes, da concorrência e das grandes mudanças da gerência. Rio de Janeiro: Campus, 1994. 189p.

MIRANDA, A. A evolução do conceito de redes automatizadas de acesso a informação e ao documento primário: o caso do SPA. In: CONGRESSO LATINO-AMERICANO DE BIBLIOTECONOMIA E DOCUMENTAÇÃO, 2. CONGRESSO BRASILEIRO DE BIBLIOTECONOMIA E DOCUMENTAÇÃO, 17. Anais. Belo Horizonte: ABMG/Esc. Bibl. UFMG, 1994. p. 294-303

OLIVEIRA, M. C. G. Informação Tecnológica em Pernambuco: acertos e desacertos. In: CONGRESSO LATINO-AMERICANO DE BIBLIOTECONOMIA E DOCUMENTAÇÃ O, 2. CONGRESSO BRASILEIRO DE BIBLIOTECONOMIA E DOCUMENTAÇÃO, 17. Anais. Belo Horizonte: ABMG/Esc. Bibl. UFMG, 1994. p. 183-191.

OLIVEIRA, P. de. Rede de informações sobre controle biológico. In: NARDO, E. A. D. de, CAPALBO, D. M. F., OLIVEIRA, M. C. B. de, MORAES, G. J. de. eds. Análise de risco, avaliação de impacto ambiental decorrente do uso de agente de controle biológico: memória do workshop. Jaguariúna: EMBRAPA/CNPMA, 1995. 127p. p.24-27. (EMBRAPA/CNPMA. Documentos).

THE TECHNOLOGY Payoff; a sweeping reorganization of work itself is boosting productivity. Business Week, p.37-48, June 14, 1993. (Special Report)

PORTER, M. E. Vantagem Competitiva. Rio de Janeiro: Campus, 1992. 512p.

SEGURANÇA Alimentar: uma abordagem do Agrobusiness. São Paulo: ABAG, 1993. $160 \mathrm{p}$.

SIMON, H. A. The Structure of II Structured Problems. Artificial Intelligence, p.181201, 1973.

SOBRINHO, F. G. Projeto Sistema Nacional 
para a Pesquisa Agropecuária. Brasília: EMBRAPA/DE, 1991. 24p.

SUNDGREN, B. Theory of database. New York: Mason/Charger, 1975. 280p.

VASCONCELOS, R. M. A. de G. e. Avaliação dos serviços informacionais. p.3-14. In: CONGRESSO LATINO-AMERICANO DE BIBLIOTECONOMIA E DOCUMENTAÇÃO, 2. CONGRESSO BRASILEIRO DE BIBLIOTECONOMIA E DOCUMENTAÇÃO, 17. Anais. Belo Horizonte: ABMG/Esc. Bibl. UFMG, 1994. p. 3-14

YEH, R., KNOX, W. An integrated approach to Business Process Reengineering. Texas: International Software Systems, Inc., 1994. $8 \mathrm{p}$.

YEH, R. T., NAUMANN, D. A., MITTERMEIR, T. T. et alii. A commonsense management model. IEEE Software, p.23-33, Nov. 1991.

\section{Nilce Chaves Gattaz}

Coordenadora do Projeto Sistema de Informação do Centro Nacional de Pesquisa de Monitoramento Ambiental na Agricultura CNPMA/EMBRAPA, M.Sc. em Ciência de Informação pela Universidade de Maryland, EUA, com área de concentração em Sistemas de Informação.

\section{Rejane Gontow}

Coordenadora do Projeto Serviço de Informação e Assistência Técnica à Agroindústria de Alimentos do Centro Nacional de Pesquisa de Tecnologia Agroindustrial de Alimentos - CTAA/ EMBRAPA, M.Sc. em Ciência da Informação pela UFRJ.

Title

A New Information System Model for R\&D Institutions

\begin{abstract}
s
A new information system approach, for $R \& D$ institutions concurrently with a paradigm change, is presented. As a way of monitoring and answering the information demand, this work focuses on the interaction and integration with the different segments of society. A methodology for implementation is presented as well as the information system model being developed at CNPMA/EMBRAPA. The gathering and dissemination of information in real time with the participation of respective information generation is emphasized.
\end{abstract}

\section{Keywords}

Information System; Data Communication Network; R\&D Institutions; Data Bases; Electronic Mail; Groups of Interest; Production Chain.

Apresentado no COBIBiii em agosto de 1995 